PROCEEDINGS OF THE

AMERICAN MATHEMATICAL SOCIETY

Volume 130, Number 1, Pages 259-266

S 0002-9939(01)06369-9

Article electronically published on July 31, 2001

\title{
THE STABLE SIGNATURE OF A REGULAR CYCLIC ACTION
}

\author{
ROBERT D. LITTLE
}

(Communicated by Ralph Cohen)

\begin{abstract}
Let $p$ be an odd prime and $g: M^{2 n} \longrightarrow M^{2 n}$ a smooth map of order $p$. Suppose that the cyclic action defined by $g$ is regular and has fixed point set $F$. If the $g$-signature $\operatorname{Sign}(g, M)$ is a rational integer and $n<p-1$, then there exists a choice of orientations such that $\operatorname{Sign}(g, M)=\operatorname{Sign} F$.
\end{abstract}

\section{INTRODUCTION}

Let $M^{2 n}$ be a smooth, closed, orientable $2 n-$ manifold. If $p$ is an odd prime, let $G_{p}$ denote the cyclic group of order $p$. Suppose that $M^{2 n}$ admits a smooth $G_{p}$ action with fixed point set $F$ and suppose that an orientation is chosen for $M^{2 n}$. We will say that $F$ is canonically oriented if $F$ is oriented so that the orientation of $F$ followed by the orientation of fibre of the normal bundle of $F$ given by the $G_{p}$ action is equal to the orientation of $M^{2 n}$ restricted to the tubular neighborhood of $F$. Throughout this paper, we will assume without explicitly stating it that if $M^{2 n}$ admits a $G_{p}$ action, then an orientation is chosen for $M^{2 n}$ and $F$ is canonically oriented. Let $g$ be a generator of $G_{p}$ and let $\operatorname{Sign}(g, M)$ be the $g$-signature of the action [2]. The $g$-signature is an algebraic integer. The action is regular if over every point in $F$, the action of $G_{p}$ on the fibre of the normal bundle of $F$ is a multiple of a fixed irreducible representation of $G_{p}$. Our first theorem asserts that if the $g$-signature of a regular action is a rational integer, then it is equal to the signature of $F$ if $p$ is sufficiently large.

Theorem A. Suppose that $M^{2 n}$ admits a regular $G_{p}$ action. If $\operatorname{Sign}(g, M)$ is a rational integer and $n<p-1$, then $\operatorname{Sign}(g, M)=\operatorname{Sign} F$.

The $g$-signature is a rational integer if the intersection form which underlies the $g$-signature is definite ([3], Lemma 1.1). Another situation in which the $g$-signature is a rational integer is when $g^{*}$ is the identity on $H^{n}(M ; \mathbb{Q})$. In this case, the $g$ signature is equal the signature of $M^{2 n}$ ([1, p. 329, 3], Section 1) and so our next theorem is an immediate consequence of Theorem A.

Theorem B. Suppose that $M^{2 n}$ admits a regular $G_{p}$ action. If $g^{*}$ is the identity on $H^{n}(M ; \mathbb{Q})$ and $n<p-1$, then $\operatorname{Sign} M=\operatorname{Sign} F$.

We now turn to the relation of our results to earlier work. If $M^{2 n}$ admits any $G_{p}$ action such that $g^{*}$ is the identity on $H^{n}(M ; \mathbb{Q})$, then $\operatorname{Sign} M \equiv \operatorname{Sign} F(\bmod$ 4) and, if the action is regular, then $\operatorname{Sign} M \equiv \operatorname{Sign} F\left(\bmod 2^{p-1}\right)([1]$, Theorems

Received by the editors May 19, 2000.

2000 Mathematics Subject Classification. Primary 57S17.

(C)2001 American Mathematical Society 
1 and 4). If $M^{2 n}$ admits a regular $G_{p}$ action and $n<p-1$, then $\operatorname{Sign} M \equiv \operatorname{Sign} F$ $(\bmod p)([\underline{6}]$, Theorem 2.2). Theorem $B$ shows that the congruence modulo the product of $2^{p-1}$ and $p$ provided by these last two results when $g^{*}$ is the identity on $H^{n}(M ; \mathbb{Q})$ and $n<p-1$ is an equality. Our methods are similar to the methods used in the proof of the former of the two results above about regular actions and we offer our own proof of it (Theorem 4.1). The techniques in the proof of the latter of these two results are quite different than ours and involve approximating regular $G_{p}$ actions by semi-free circle actions ([6], Theorem 2.1).

Our next theorem asserts that under the hypotheses of Theorem A, $\operatorname{Sign}(g, M)=$ Sign $F=0$ if the dimension of $F$ is small.

Theorem C. Suppose that $M^{2 n}$ admits a regular $G_{p}$ action. If $\operatorname{Sign}(g, M)$ is a rational integer and $n<p-1$ and the dimensions of the components of $F$ are all strictly less than $n$, then $\operatorname{Sign}(g, M)=\operatorname{Sign} F=0$.

Theorem D. Suppose that $M^{2 n}$ admits a regular $G_{p}$ action. If $\operatorname{Sign}(g, M)$ is a nonzero rational integer and $n<p-1$, then $n$ is even and $F$ contains a nonempty component of dimension at least $n$.

Theorem E. Suppose that $M^{2 n}$ admits a regular $G_{p}$ action. If $g^{*}$ is the identity on $H^{n}(M ; \mathbb{Q})$ and $\operatorname{Sign} M \neq 0$ and $n<p-1$, then $F$ contains a nonempty component of dimension at least $n$.

If $M^{2 n}$ admits a regular $G_{p}$ action and $n<p-1$ and Sign $M \not \equiv 0(\bmod p)$, then $F$ contains a nonempty component of dimension at least $n$ ([6], Corollary 2.7). Theorem $E$ shows that in the presence of the hypothesis that $g^{*}$ is the identity on $H^{n}(M ; \mathbb{Q})$, the condition $\operatorname{Sign} M \not \equiv 0(\bmod p)$ can be weakened to $\operatorname{Sign} M \neq 0$ to conclude that $F$ contains a nonempty component of dimension at least $n$ if $n<p-1$. If $I_{2 n}(p)$ is the subgroup of the oriented bordism group $\Omega_{2 n}$ consisting of those bordism classes all of whose Pontrjagin numbers are divisible by $p$ and if $[M] \neq 0$ in $\Omega_{2 n} / I_{2 n}(p)$, then any regular $G_{p}$ action on $M^{2 n}$ contains a fixed component of dimension at least $n$ ([8], Theorem 1.3).

Our main tool in the proofs of the above theorems is the Atiyah-Singer $g$ Signature Formula for regular $G_{p}$ actions in the formulation of Berend and Katz ([2], 3], Theorem 2.2). This form of the signature formula expresses the contribution to the $g$-signature of the normal bundle of each slice type as an element of the ring $\mathbb{Z}\left[\alpha_{1}, \alpha_{2}, \ldots, \alpha_{p-1}\right]$, where $\alpha_{j}=\left(\lambda^{j}+1\right)\left(\lambda^{j}-1\right)^{-1}, 1 \leq j \leq p-1$, with $\lambda=\exp (2 \pi i / p)$. Each $\alpha_{j}$ is an algebraic number with a polynomial of degree $p-1$ as minimal polynomial over $\mathbb{Q}$. The Berend-Katz form of the $g$-signature formula is therefore very useful in the study of the effect on the $g$-signature of the stabilization inequality $n<p-1$.

This paper is organized as follows. Section 2 contains a discussion of the AtiyahSinger $g$-Signature Formula $(A S g S F)$ and a version of this formula for regular actions. In Section 3, we discuss the algebraic numbers $\alpha_{j}, 1 \leq j \leq p-1$, and present the elementary properties of these numbers which we will use in the proofs of the above theorems. Section 4 contains the proofs of Theorems A and C and, consequently, the proofs of Theorems B, D and E. We also present the promised proof of the congruence mod $2^{p-1}$ ([1], Theorem 4). 


\section{The Atiyah-Singer $g$-Signature for Regular $G_{p}$ aCtions}

Suppose $M^{2 n}$ admits an arbitrary smooth $G_{p}$ action with generator $g$ and let $\nu$ be the normal bundle of $F$ in $M^{2 n}$. Over each connected component of $F, \nu$ splits into a direct sum of eigenbundles $\nu_{j}$ where $\nu_{j}$ is a complex bundle of complex dimension $m_{j}$ and $g$ acts on $\nu_{j}$ as multiplication by $\lambda^{j}, \lambda=\exp (2 \pi i / p)$ ([3] , Section 2, [6], p. 183). In this fashion, we have associated with each connected component of $F$ a normal slice type, a $(p-1)$-tuple of nonnegative integers $m=\left(m_{1}, m_{2}, \ldots, m_{p-1}\right)$, where $m_{j}=\operatorname{dim}_{\mathbb{C}} \nu_{j}, 1 \leq j \leq p-1$. Let $F_{m}$ be the union of all connected components with slice type $m$ and let $\nu_{m}$ be the normal bundle of $F_{m}$ in $M^{2 n}$. Note that if $m=\left(m_{1}, m_{2}, \ldots, m_{p-1}\right)$, then $\operatorname{dim}_{\mathbb{C}} \nu_{m}=\sum_{j=1}^{p-1} m_{j}$.

We need three more definitions. Let $x_{j, \ell} \in H^{2}\left(F_{m} ; \mathbb{Z}\right), 1 \leq j \leq p-1,1 \leq \ell \leq$ $m_{j}$, be the $\mathrm{Wu}$ classes of $\nu_{m}$, i.e. the elementary symmetric polynomials in $x_{j, \ell}$, $1 \leq \ell \leq m_{j}$, are the Chern classes of $\nu_{j}$. Let $L\left(F_{m}\right)$ be the Hirzebruch $L$-class of $F_{m}$ and let $\left[F_{m}\right]$ be the fundamental orientation class of $F_{m}$ determined by the canonical orientation of $F_{m}$.

Theorem 2.1 (Atiyah-Singer $g$-Signature Formula [2]). Let $M^{2 n}$ be a smooth, closed, oriented $2 n$-manifold and let $g: M^{2 n} \longrightarrow M^{2 n}$ be a diffeomorphism of order $p$. If $F$ is canonically oriented, then

$$
\operatorname{Sign}(g, M)=\sum_{m} \prod_{j} \prod_{\ell=1}^{m j} \frac{\lambda^{j} e^{2 x_{j, \ell}}+1}{\lambda^{j} e^{2 x_{j, \ell}}-1} L\left(F_{m}\right)\left[F_{m}\right] .
$$

The double product inside the summation sign is the contribution of $\nu_{m}$ to the $g$-signature and is denoted by Cont $\nu_{m}$. Berend and Katz have developed an alternative expression for Cont $\nu_{m}$ and the $A S g S F$ which we will state after some technical preparation.

Let $\mathbb{Z}_{+}$denote the set of nonnegative integers. If $q$ is a positive integer, let $S(q)$ denote the symmetric group on $q$ letters and, given a slice type $m=\left(m_{1}, m_{2}, \ldots\right.$, $\left.m_{p-1}\right)$, let $S(m)=\prod_{j} S\left(m_{j}\right), 1 \leq j \leq p-1$. Note that $S(m)$ acts naturally on $\widetilde{\Omega}(m)=\prod_{j} \mathbb{Z}_{+}^{m j}, 1 \leq j \leq p-1$, and put $\Omega(m)=\widetilde{\Omega}(m) / S(m)$. The set $\Omega(m)$ admits four useful functions ([3], Section 2). The $j-$ norm, $\|\cdot\|_{j}: \Omega(m) \longrightarrow \mathbb{Z}_{+}$, simply sums the entries in an element of $\Omega(m)$ which come from $\mathbb{Z}_{+}^{m_{j}}$, the $j_{-}$ counter, $|\cdot|_{j}: \Omega(m) \longrightarrow \mathbb{Z}_{+}$, counts the number of nonzero entries in an element of $\Omega(m)$ coming from $\mathbb{Z}_{+}^{m_{j}}$, and the total norm and total counter are defined by $\|\cdot\|=\sum_{j}\|\cdot\|_{j}$ and $|\cdot|=\sum_{j}|\cdot|_{j}$. Let $\alpha_{j}=\left(\lambda^{j}+1\right)\left(\lambda^{j}-1\right)^{-1}, 1 \leq j \leq p-1$, where $\lambda=\exp (2 \pi i / p)$ as above.

Theorem 2.3 (3), Theorem 2.2). Let $M^{2 n}$ be a smooth, closed, oriented $2 n-m a n i-$ fold and let $g: M^{2 n} \longrightarrow M^{2 n}$ be a diffeomorphism of order $p$. There exist rational integers $S_{\omega}\left(\nu_{m}\right), \omega \in \Omega(m)$, for every normal slice type $m$, with the property that if $F$ is canonically oriented, then we have

$$
\operatorname{Sign}(g, M)=\sum_{m} \sum_{\omega \in \Omega(m)}(-1)^{|| \omega||}\left(\prod_{j} \alpha_{j}^{m_{j}+|| \omega||_{j}-2|\omega|_{j}}\left(\alpha_{j}^{2}-1\right)^{|\omega|_{j}}\right) S_{\omega}\left(\nu_{m}\right) .
$$

Theorems 2.1 and 2.3 are special cases of the Aitiyah-Singer $g$-Signature Theorem which is valid for diffeomorphisms of arbitrary order if it is assumed that for 
each slice type $m, \nu_{m}$ admits an equivariant complex structure. This condition is automatically satisfied if the order of the diffeomorphism is odd.

The inner summation in (2.4), the summation over all $\omega \in \Omega(m)$ for a given normal slice type $m$, is the Berend-Katz formula for Cont $\nu_{m}$. Formula (2.4) has proven to be preferable to (2.2) for some applications. Formula (2.4) in the case where $\nu_{m}$ is a complex line bundle has been used to study $G_{p}$ actions on complex projective $n$-space which fix an isolated point (4], Formula (4.5), 7], Formula $(1.3))$.

The integers $S_{\omega}\left(\nu_{m}\right)$ can be described as follows. If $m=\left(m_{1}, m_{2}, \ldots, m_{p-1}\right)$ is a normal slice type and $x_{j, \ell} \in H^{2}\left(F_{m} ; \mathbb{Z}\right)$ is a Wu class, let $Y_{j, \ell} \subset F_{m}$ be the codimension-2 submanifold of $F_{m}$ which is the Poincaré dual of $x_{j, \ell}$. If $\widetilde{\omega}=\left(\omega_{j, \ell}\right) \in$ $\widetilde{\Omega}(m)$, put $Y^{(\widetilde{\omega})}=\pitchfork_{j, \ell} Y_{j, \ell}^{\left(\omega_{j, \ell}\right)}$, i.e. $Y^{(\widetilde{\omega})}$ is obtained by taking the transverse selfintersection of $\omega_{j, \ell}$ copies of $Y_{j, \ell}$ with itself, namely $Y_{j, \ell}^{\left(\omega_{j, \ell}\right)}$, and then taking the transversal intersection of this family of submanifolds over all $j$ and $\ell, 1 \leq j \leq p-1$, $1 \leq \ell \leq m_{j}$. If $\omega$ is the image of $\widetilde{\omega}$ in $\Omega(m)$, then

$$
S_{\omega}\left(\nu_{m}\right)=\sum_{\sigma \in S(m)}\left|S t_{\widetilde{\omega}}\right|^{-1} \operatorname{Sign} Y^{(\sigma \widetilde{\omega})},
$$

where $\left|S t_{\widetilde{\omega}}\right|$ denotes the order of the stabilizer of $\widetilde{\omega}$ ([3], Section 2). These integervalued quasi-signatures are tractable in some cases. For example, if $F_{m}$ has complex codimension one, i.e. $m=\left(m_{1}, m_{2}, \ldots, m_{p-1}\right)$ has the form $m_{j_{0}}=1$ for some $j_{0}$, $1 \leq j_{0} \leq p-1, m_{j}=0, j \neq j_{0}$, then the quasi-signatures are the signatures of the self-intersections of $F_{m}$ with itself and (2.4) is fairly simple ([7], Formula (1.3)). We will see that (2.4) can be handled if the action is regular. We begin with a definition of regularity in terms of normal slice types.

Definition 2.6. A $G_{p}$ action on $M^{2 n}$ is regular if there exists a $j_{0}, 1 \leq j_{0} \leq p-1$, such that for every slice type $m=\left(m_{1}, m_{2}, \ldots, m_{p-1}\right), m_{j}=0$ if $j \neq j_{0}$.

Note that if $m=\left(m_{1}, m_{2}, \ldots, m_{p-1}\right)$ is a normal slice type of a regular $G_{p}$ action, and $m_{j}=0$ if $j \neq j_{0}$, then $m_{j_{0}}$ is the complex codimension of $F_{m}$ in $M^{2 n}$. If the regular action has $s$ normal slice types, then each one is associated with a complex codimension $c_{i}, 1 \leq i \leq s$, i.e. the normal bundles at the slice types are bundles of complex dimension $c_{i}$. We will identify a normal slice of a regular action with its complex codimension. Let $F^{2 n-2 c}$ be the union of all connected components of $F$ with codimension $2 c$ and let $\nu_{c}$ be its normal bundle if $c \in\left\{c_{1}, c_{2}, \ldots, c_{s}\right\}$, $0 \leq c \leq n$.

We now begin to examine (2.4) in the special case of a regular action. Let $m$ be a normal slice type of a regular $G_{p}$ action with codimension $c$, i.e. $m_{j_{0}}=c$ and $m_{j}=0, j \neq j_{0}$. Note that in (2.4), $\Omega(m)=\mathbb{Z}_{+}^{c} / S(c)$ if $c \neq 0$ and the product inside the inner sum is just a single term at $j=j_{0}$. The $j_{0}-$ norm and the $j_{0}-$ counter reduce to the total norm and total counter, i.e. $\|\cdot\|=\|\cdot\|_{j_{0}}$ and $|\cdot|=|\cdot|_{j_{0}}$. The range of $\|\omega\|$ for $\omega \in \mathbb{Z}_{+}^{c} / S(c)$ is $0 \leq\|\omega\| \leq n-c$. To see this, note that if $\widetilde{\omega} \in \mathbb{Z}_{+}^{c}$ covers $\omega$, then the real dimension of the submanifold $Y^{(\widetilde{\omega})}$ is $2(n-c-\|\omega\|)$ and so $S_{\omega}\left(\nu_{c}\right)=0$ unless $0 \leq\|\omega\| \leq n-c$. If $\omega \in \mathbb{Z}_{+}^{c} / S(c)$ and $|\omega|=j$, where $0 \leq j \leq c$, then $j \leq\|\omega\| \leq n-c$. With these preliminaries, we are ready to define a useful combination of the quasi-signatures of $\nu_{c}$ and then state and prove a theorem about (2.4) in the special case of a regular $G_{p}$ action. 
Definition 2.7. Suppose that $M^{2 n}$ admits a regular $G_{p}$ action with the complex codimensions of the components of $F$ belonging to the set $\left\{c_{1}, c_{2}, \ldots, c_{s}\right\}$. If $c \in$ $\left\{c_{1}, c_{2}, \ldots, c_{s}\right\}, 1 \leq j \leq c, j \leq k \leq n-c$, then let $s(c, j, k)=\left\{\omega \in \mathbb{Z}_{+}^{c} / S(c):|\omega|=\right.$ $j,\|\omega\|=k\}$ and put

$$
S_{c}(j, k)\left(\nu_{c}\right)=\sum_{\omega \in s(c, j, k)} S_{\omega}\left(\nu_{c}\right) .
$$

Formula (2.8) gathers together those quasi-signatures of $\nu_{c}$ with the property that $\omega$ has $j$ nonzero entries and the sum of the entries in $\omega$ is $k$. This helps write (2.4) in the special case of a regular $G_{p}$ action as an ordinary double sum.

Theorem 2.9 (Berend-Katz $A S g S F$ for regular $G_{p}$ actions). Suppose that $M^{2 n}$ admits a regular $G_{p}$ action with the complex codimensions of $F$ belonging to the set $\left\{c_{1}, c_{2}, \ldots, c_{s}\right\}$. There exists an $\alpha \in\left\{\alpha_{1}, \alpha_{2}, \ldots, \alpha_{p-1}\right\}$ such that for every $c \in\left\{c_{1}, c_{2}, \ldots, c_{s}\right\}$, we have

$$
\text { Cont } \nu_{c}=\operatorname{Sign} F^{2 n-2 c} \alpha^{c}+\sum_{j=1}^{c} \sum_{k=j}^{n-c}(-1)^{k} \alpha^{c+k-2 j}\left(\alpha^{2}-1\right)^{j} S_{c}(j, k)\left(\nu_{c}\right) \text {. }
$$

Proof. Formula (2.10) follows from (2.4) in the case of a regular $G_{p}$ action. We know from Definition 2.6 that there exists $j_{0}, 1 \leq j_{0} \leq p-1$, such that every normal slice type $m$ is of the form $m_{j_{0}}=c, m_{j}=0, j \neq j_{0}$, where $c \in\left\{c_{1}, c_{2}, \ldots, c_{s}\right\}$. We also know that for every normal slice type $m, \Omega(m)=\mathbb{Z}_{+}^{c} / S(c)$ if $c \neq 0$, and so, if $c \in\left\{c_{1}, c_{2}, \ldots, c_{s}\right\}$, then (2.4) implies that for $\alpha=\alpha_{j_{0}}$,

$$
\text { Cont } \nu_{c}=\sum_{\omega \in \mathbb{Z}_{+}^{c} / S(c)}(-1)^{|| \omega||} \alpha^{c+|| \omega||-2|\omega|}\left(\alpha^{2}-1\right)^{|\omega|} S_{\omega}\left(\nu_{c}\right) .
$$

Formula (2.10) follows from (2.11) together with the organization provided by Definition 2.7 and the fact that if $\|\omega\|=0$, then $S_{\omega}\left(\nu_{c}\right)=\operatorname{Sign} F^{2 n-2 c}$.

The $g$-signature formula for regular $G_{p}$ actions follows from (2.10): $\operatorname{Sign}(g, M)=$ $\sum$ Cont $\nu_{c}$ where the sum is taken over all $c \in\left\{c_{1}, c_{2}, \ldots, c_{s}\right\}$. For our purposes, we will also need a statement of the $g$-signature formula which focuses on the qualitative implications of (2.10).

Corollary 2.12. Suppose that $M^{2 n}$ admits a regular $G_{p}$ action with the complex codimensions of $F$ belonging to the set $\left\{c_{1}, c_{2}, \ldots, c_{s}\right\}$, generated by a diffeomorphism $g: M^{2 n} \longrightarrow M^{2 n}$ of order $p$. There exist a polynomial $p(x)$ with integer coefficients of degree at most $n-2$ and an $\alpha \in\left\{\alpha_{1}, \alpha_{2}, \ldots, \alpha_{p-1}\right\}$ such that

$$
\operatorname{Sign}(g, M)=\sum_{i=1}^{s} \operatorname{Sign} F^{2 n-2 c_{i}} \alpha^{c_{i}}+\left(\alpha^{2}-1\right) p(\alpha) .
$$

Proof. Formula (2.13) follows from (2.4) and (2.10).

\section{The Algebraic numbers $\alpha_{j}$}

If $p$ is an odd prime and $\mu=(p-1) / 2$, the polynomial

$$
m_{p}(x)=\sum_{k=0}^{\mu}\left(\begin{array}{c}
p \\
2 k+1
\end{array}\right) x^{\mu-k}
$$

will play an important part when we turn to the proofs of the results in the introduction. 
Proposition 3.2. If $p$ is an odd prime and $1 \leq j \leq p-1$, then $m_{p}\left(\alpha_{j}^{2}\right)=0$.

Proof. The equation $\left(\alpha_{j}+1\right)\left(\alpha_{j}-1\right)^{-1}=\lambda^{j}$ implies that $\left(\alpha_{j}+1\right)^{p}=\left(\alpha_{j}-1\right)^{p}$ and so the binomial theorem implies that $m_{p}\left(\alpha_{j}^{2}\right)=0,1 \leq j \leq p-1$.

Proposition 3.2 establishes that $\alpha_{j}, 1 \leq j \leq p-1$, is an algebraic number, and that $\alpha_{j}^{-1}$ is an algebraic integer, $1 \leq j \leq p-1$. The polynomial $m_{p}(x)$ is well known ([3], Proposition 4.5, 9], pp. 220-221). Our next corollary follows immediately from Proposition 3.2 and the fact that $m_{p}\left(x^{2}\right)$ is irreducible over $\mathbb{Q}$ by Eisenstein's Criterion.

Corollary 3.3. If $1 \leq j \leq p-1$, then $m_{p}\left(x^{2}\right)$ is the minimal polynomial over $\mathbb{Q}$ of $\alpha_{j}$. In particular, $\left[\mathbb{Q}\left(\alpha_{j}\right): \mathbb{Q}\right]=p-1$.

Lemma 3.4. If $a(x)=\sum_{k=0}^{q} a_{k} x^{k}$ and $b(x)=\sum_{k=0}^{q-p+1} b_{k} x^{k}$ are rational polynomials where $q \geq p-1$ and $a(x)=m_{p}\left(x^{2}\right) b(x)$, then

$$
\sum_{k=0}^{q} a_{k}=2^{p-1} \sum_{k=0}^{q-p+1} b_{k} .
$$

If $a_{k} \in \mathbb{Z}, 0 \leq k \leq q-p+1$, then $b_{k} \in \mathbb{Z}, 0 \leq k \leq q-p+1$, and $a_{k} \in \mathbb{Z}$, $q-p+2 \leq k \leq q$. In particular, if $a_{k} \in \mathbb{Z}, 0 \leq k \leq q-p+1$, then $a_{k} \in \mathbb{Z}$, $0 \leq k \leq q$, and

$$
\sum_{k=0}^{q} a_{k} \equiv 0\left(\bmod 2^{p-1}\right) .
$$

Proof. Formula (3.5) follows because $m_{p}(1)=2^{p-1}$. The statement about the integrality of the coefficients of $b(x)$ is an elementary consequence of the fact that the constant term of $m_{p}(x)$ is one together with induction and (3.6) is the sum of the integrality statement and (3.5).

We will really only need two elementary properties of the algebraic numbers $\alpha_{j}$, $1 \leq j \leq p-1$ : they are of degree $p-1$ over $\mathbb{Q}$ by Corollary 3.3 and if $a(x)$ is a polynomial with integer coefficients such that $a\left(\alpha_{j}\right)=0$ for some $j, 1 \leq j \leq p-1$, then $m_{p}\left(x^{2}\right)$ divides $a(x)$ by Corollary 3.3 and so $a(1) \equiv 0\left(\bmod 2^{p-1}\right)$ by $(3.6)$. The numbers $\alpha_{j}$ have many interesting properties. For example there is a family of summation identities which contain as a special case the sum of $\alpha_{j}^{2}, 1 \leq j \leq p-1$ ([5], (3) p. 100).

\section{The proofs of Theorems A And C}

Throughout this section, we will assume that $M^{2 n}$ admits a $G_{p}$ action with generator $g$. We begin by retrieving a signature congruence from [1].

Theorem 4.1 ([1], Theorem 4). If $M^{2 n}$ admits a regular $G_{p}$ action and $\operatorname{Sign}(g, M)$ is a rational integer, then $\operatorname{Sign}(g, M) \equiv \operatorname{Sign} F\left(\bmod 2^{p-1}\right)$. In particular, if $g^{*}$ is the identity on $H^{n}(M ; \mathbb{Q})$, then $\operatorname{Sign} M \equiv \operatorname{Sign} F\left(\bmod 2^{p-1}\right)$.

Proof. Suppose that the complex codimensions of $F$ belong to the set $\left\{c_{1}, c_{2}, \ldots, c_{s}\right\}$ and put

$$
a(x)=\sum_{i=1}^{s} \operatorname{Sign} F^{2 n-2 c_{i}} x^{c_{i}}+\left(x^{2}-1\right) p(x)-\operatorname{Sign}(g, M)
$$


where $p(x)$ is the polynomial of Corollary 2.12. There is an $\alpha \in\left\{\alpha_{1}, \alpha_{2}, \ldots, \alpha_{p-1}\right\}$ such that $a(\alpha)=0$, and so if $\operatorname{Sign}(g, M)$ is a rational integer, then $a(1) \equiv 0(\bmod$ $\left.2^{p-1}\right)$ by (3.6) if the degree of $a(x)$ is at least $p-1$ and because $a(x)$ is identically zero otherwise. This is the first statement in Theorem 4.1. The second statement follows from the first $\operatorname{since} \operatorname{Sign}(g, M)=\operatorname{Sign} M$ if $g^{*}$ is the identity on $H^{n}(M ; \mathbb{Q})$ (1], p. 329, [3, Section 1).

We now turn to the proof of Theorem A and, as a consequence, Theorem B. These two results were stated separately in the introduction to emphasize the fact that the result stated in Theorem B is related to two results in the literature, one similar to Theorem A ([1], Theorem 4) and another from a different area ([6], Theorem 2.2). Our next result is a strengthened version of Theorems A and B. Let $F_{\text {even }}$ be the union of all components of $F$ with even complex codimension and let $F_{\text {odd }}$ be the union of all components of $F$ with odd complex codimension.

Theorem 4.3. If $M^{2 n}$ admits a regular $G_{p}$ action and $\operatorname{Sign}(g, M)$ is a rational integer and $n<p-1$, then $\operatorname{Sign}(g, M)=\operatorname{Sign} F_{\text {even }}$ and $\operatorname{Sign} F_{\text {odd }}=0$ and so $\operatorname{Sign}(g, M)=\operatorname{Sign} F$. In particular, if $g^{*}$ is the identity on $H^{n}(M ; \mathbb{Q})$ and $n<p-1$, then $\operatorname{Sign} M=\operatorname{Sign} F_{\text {even }}=\operatorname{Sign} F$.

Proof. Suppose that the complex codimensions of $F$ belong to the set $\left\{c_{1}, c_{2}, \ldots, c_{s}\right\}$ and let $a(x)$ be the polynomial defined by (4.2). There is an $\alpha \in\left\{\alpha_{1}, \alpha_{2}, \ldots, \alpha_{p-1}\right\}$ such that $a(\alpha)=0$ and so if $\operatorname{Sign}(g, M)$ is a rational integer and $n<p-1$, then $a(x)$ is identically zero in view of Corollary 3.3 and the fact that the degree of $a(x)$ is at most $n$. Therefore $a( \pm 1)=0$ and so the equations $\operatorname{Sign}(g, M)=\operatorname{Sign} F_{\text {even }}$ and $\operatorname{Sign} F_{\text {odd }}=0$ follow from (4.2). If $g^{*}$ is the identity on $H^{n}(M, \mathbb{Q})$, then $\operatorname{Sign}(g, M)=\operatorname{Sign} M$ and so $\operatorname{Sign} M=\operatorname{Sign} F_{\text {even }}=\operatorname{Sign} F$ if $n<p-1$.

Our next step is the proof of Theorems C, D and E. Our strategy will be to prove Theorem $\mathrm{C}$ and then combine Theorems D and E into a single easy Corollary of Theorem C. Theorem E was singled out for emphasis because of related results ([6], Theorem 2.2). Our next theorem is equivalent to Theorem $\mathrm{C}$ and the one following it is equivalent to Theorems $\mathrm{D}$ and $\mathrm{E}$.

Theorem 4.4. If $M^{2 n}$ admits a regular $G_{p}$ action and $\operatorname{Sign}(g, M)$ is a rational integer and $n<p-1$ and the dimensions of the components of $F$ are all strictly less than $n$, then $\operatorname{Sign}(g, M)=\operatorname{Sign} F=0$.

Proof. Suppose that the complex codimensions of $F$ belong to the set $\left\{c_{1}, c_{2}, \ldots, c_{s}\right\}$. It follows from (2.4) and Theorem 2.9 that for some $\alpha \in\left\{\alpha_{1}, \alpha_{2}, \ldots, \alpha_{p-1}\right\}$,

$$
\operatorname{Sign}(g, M)=\sum_{i=1}^{s} \operatorname{Cont} \nu_{c_{i}},
$$

where each Cont $\nu_{c_{i}}$ is given by (2.10). Now suppose that the dimensions of the components of $F$ are all strictly less than $n$, i.e. $2 c_{i}>n, 1 \leq i \leq s$, and that, for the moment, no restrictions are placed on $\operatorname{Sign}(g, M)$ and the size of $n$. We assert that every exponent of $\alpha$ in the right hand side of (4.5) is positive. Deny. Then there is a $c \in\left\{c_{1}, c_{2}, \ldots, c_{s}\right\}$ such that $\alpha$ appears to the power zero in Cont $\nu_{c}$ as given by (2.10). This means that either $c=0$ or there exist integers $j$ and $k$, $1 \leq j \leq c, j \leq k \leq n-c$, such that $c+k-2 j=0$ or $k=2 j-c$. The former is impossible because $2 c>n$. The latter is also impossible because $k=2 j-c$ means $j \leq 2 j-c \leq n-c$ and so $c+j \leq n$ and $c+j \leq 2 j$ which means $2 c \leq n$ and 
this again contradicts $2 c>n$. Now suppose that $\operatorname{Sign}(g, M)$ is a rational integer. It follows from (4.5) and what we just proved about the exponents of $\alpha$ in (4.5) that $\operatorname{Sign}(g, M)$ is the constant term in an integral polynomial with $\alpha$ as a zero. If $n<p-1$ is added to the list of hypotheses, then $\operatorname{Sign}(g, M)=0$ in view of Corollary 3.3 and the fact that the degree of the polynomial in $\alpha$ in (4.5) is at most $n$. Therefore $\operatorname{Sign}(g, M)=\operatorname{Sign} F=0$ by Theorem 4.3.

Theorem 4.6. If $M^{2 n}$ admits a regular $G_{p}$ action and $\operatorname{Sign}(g, M)$ is a nonzero rational integer and $n<p-1$, then $n$ is even and $F$ contains a nonempty component of dimension at least $n$. In particular, if $g^{*}$ is the identity on $H^{n}(M, \mathbb{Q})$ and Sign $M \neq 0$ and $n<p-1$, then $F$ contains a nonempty component of dimension at least $n$.

Proof. If $\operatorname{Sign}(g, M)$ is a rational integer and $n<p-1$, then $\operatorname{Sign}(g, M)=\operatorname{Sign} F_{\text {even }}$ by Theorem 4.3 and so $n$ is even if $\operatorname{Sign}(g, M)$ is a nonzero rational integer and $n<p-1$. If $\operatorname{Sign}(g, M)$ is a nonzero rational integer and $n<p-1$, then it follows from Theorem 4.4 that $F$ contains a nonempty component of dimension at least $n$. If $g^{*}$ is the identity on $H^{n}(M, \mathbb{Q})$, then $\operatorname{Sign}(g, M)=\operatorname{Sign} M$ and so, if $\operatorname{Sign} M \neq 0$ and $n<p-1$, then $F$ contains a nonempty component of dimension at least $n$ by Theorem 4.4.

\section{REFERENCES}

1. J. P. Alexander, G. C. Hamrick and J. W. Vick, The signature of the fixed set of odd period, Proc. Amer. Math. Soc. 57 (1976), 327-331. MR 53:11632

2. M. F. Atiyah and I. M. Singer, The index of elliptic operators, III, Ann. of Math. 87 (1968), 546-604. MR 38:5245

3. D. Berend and G. Katz, Separating topology and number theory in the Atiyah-Singer gsignature formula, Duke Math. J. 61 (1990), 939-971. MR 91k:58122

4. K. H. Dovermann and R. D. Little, Cohomology complex projective space with degree one codimension-two fixed submanifolds, Pacific J. Math. 173 (1996), 197-211. MR 97f:57039

5. F. Hirzebruch and D. Zagier, The Atiyah-Singer Theorem and Elementary Number Theory, Mathematics Lecture Series No. 3 (1974), Publish or Perish, Boston. MR 58:31291

6. K. Kawakubo, The index and the generalized Todd genus of $\mathbb{Z}_{p}$ actions, Amer. J. Math. 97 (1975), 182-204. MR 51:14118

7. R. D. Little, Self-intersection of fixed manifolds and relations for the multisignature, Math. Scand. 69 (1991), 167-178. MR 93a:57030

8. D. C. Royster, An analogue of the stabilization map for regular $\mathbb{Z}_{p}$ actions, Rocky Mountain J. Math. 24 (1994), 689-708. MR 95h:57044

9. C. T. C. Wall, Surgery on Compact Manifolds, Academic Press, London (1970). MR 55:4217

Department of Mathematics, University of Hawail, Honolulu, Hawair 96822-2330

E-mail address: little@math.hawaii.edu 